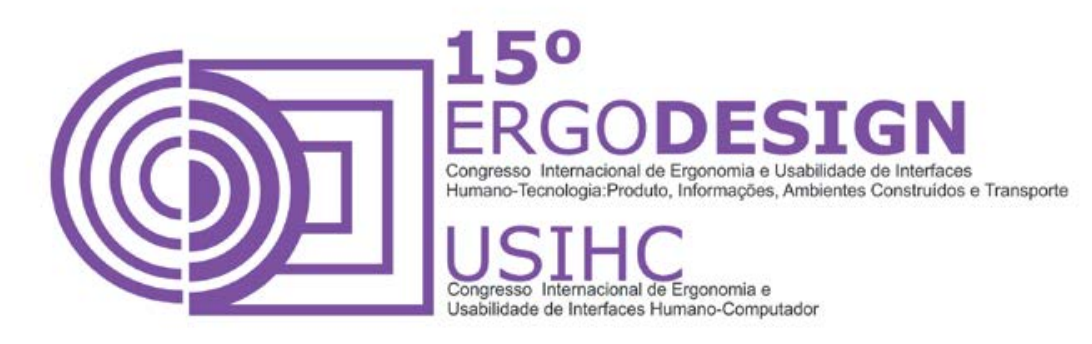

\title{
APLICAÇÃO DA TÉCNICA CARDSORTING PARA REFORMULAÇÃO DO MENU DO SITE DO INSTITUTO DO CÉREBRO DA UFRN
}

\section{CARDSORTING TECHNIQUE: REFORMULATION OF SITE'S MENU OF INSTITUTO DO CÉREBRO DA UFRN}

\author{
SILVA, Gabriel Marcos (1); \\ SANTA ROSA, José Guilherme (2) \\ (1) Universidade Federal do Rio Grande do Norte, Aluno do Mestrado Profissional em Design \\ e-mail:gabriel.fip@gmail.com \\ (2) Universidade Federal do Rio Grande do Norte, Doutor \\ e-mail:jguilhermesantarosa@gmail.com
}

\begin{abstract}
RESUMO
Este trabalho apresenta os resultados da aplicação do método Cardsorting para reformulação do menu do site do Instituto do Cérebro da Universidade Federal do Rio Grande do Norte. A técnica Cardsorting, aplicada com um grupo de seis sujeitos do público-alvo da Instituição, foi empregada para levantar informações a respeito de como os usuários do site categorizam as informações disponíveis num processo de busca, identificando qual terminologia é a mais usual. Concluiu-se, por tanto, que foram verificados, dentre outros, vários problemas relacionados à rotulagem e posicionamento de alguns termos do menu.

Palavras-chave: Arquitetura da Informação; Usabilidade; Cardsorting.
\end{abstract}




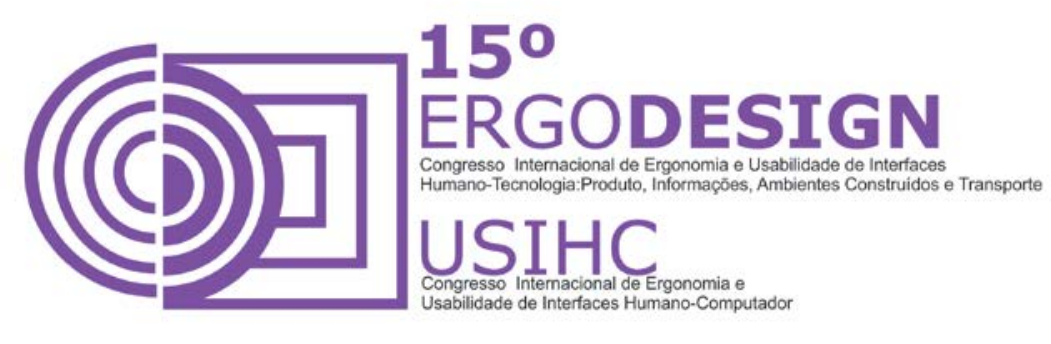

\begin{abstract}
This paper presents the results of applying the method Cardsorting to reformulation of site menu of the Brain Institute of the Federal University of Rio Grande do Norte. The Cardsorting method, applied with a group of six users of the target audience of the institution, was used to gather information about how site users categorize the information available in a search process, identifying which terminology is the most common. It was concluded, therefore, that were checked, among others, several problems related to labeling and positioning of some terms of the menu.
\end{abstract}

Keywords: Information Architeture; Usabilit; Cardsorting.

\title{
1. INTRODUÇÃO
}

Nos últimos cinco anos, vários pesquisadores brasileiros e estrangeiros com treinamento em importantes centros de pesquisa dos EUA e da Europa se radicaram em Natal e construíram o Instituto do Cérebro da Universidade Federal do Rio Grande do Norte - UFRN (ICE, 2011).

O Instituto do Cérebro se concretizou na UFRN com grande apoio do MEC e do MCTI, e sedia o Programa de Pós-Graduação em Neurociências da UFRN em níveis de mestrado e doutorado, e tem como objetivo contribuir para a formação multidisciplinar dos alunos de graduação da UFRN. Destaca-se, ainda, que o Instituto do Cérebro também desenvolve o eixo de Neurociências no âmbito do Bacharelado em Ciência e Tecnologia na Escola de Ciência e Tecnologia da UFRN (ICE, 2011).

As pesquisas desenvolvidas por Professores e alunos são constantemente publicadas em periódicos de grande circulação internacional. As linhas dessas pesquisas são bastante abrangentes, e estão relacionadas à dinâmica do sistema visual, conexões sensorimotoras, neurobiologia celular, oscilações neurais, comunicação animal, papel cognitivo do sono, modelos computacionais de circuitos neurais, neuroengenharia, bem como o estudo dos mecanismos e possíveis tratamentos para epilepsia, doenças vasculares, psicoses, depressão e outros transtornos neurais.

Todas as publicações são divulgadas no site para que as pessoas possam conhecer o trabalho do Instituto do Cérebro.

Então, tendo em vista a importância do site Institucional para Instituto do Cérebro da UFRN, realizamos este estudo com foco na reformulação do menu por meio da aplicação do método Cardsorting. O site pode ser acessado a partir do endereço eletrônico http://www.neuro.ufrn.br. 


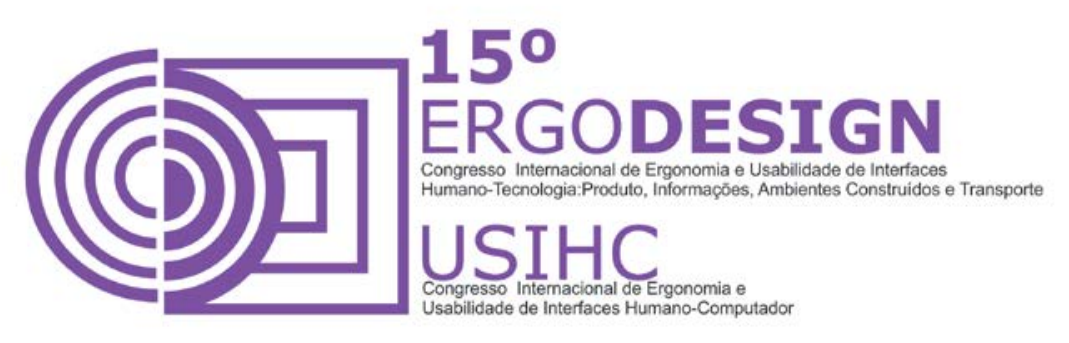

\section{REFERENCIAL TEÓRICO}

\section{$2.1 \quad$ Usabilidade}

O termo usabilidade é frequentemente usado para se referir à capacidade de um produto ser facilmente usado. De acordo com a ISO 9241, interpretada pela NBR 9241-11 (2002), usabilidade é a medida na qual um produto pode ser usado por usuários específicos para alcançar objetivos específicos com eficácia, eficiência e satisfação em um contexto específico de uso.

A usabilidade é a qualidade que caracteriza o uso dos programas e aplicações. Assim, ela não é uma qualidade intrínseca de um sistema, mas depende de um acordo entre as características de sua interface e de seus usuários ao buscarem determinados objetivos em determinadas situações de uso (CYBIS; BETIOL; FAUST, 2010, p. 16).

Para (NIELSEN, 1993), a usabilidade de um sistema está associada a cinco atributos: 1) Facilidade de aprendizagem: o aprendizado ocorre com o uso, mas o sistema deve ser fácil, para que o usuário consiga explorá-lo rapidamente. Um indicativo de que o sistema possui este atributo é quando usuários inexperientes atingem certo grau de proficiência em um curto período de tempo; 2) Eficiência: o uso do sistema deve ser eficiente, permitindo que após interagir com ele, o usuário experiente atinja um alto grau de produtividade na realização de suas tarefas; 3) Facilidade de memorização: após um período sem utilizar o sistema, 0 usuário ocasional é capaz de retornar e realizar suas tarefas sem a necessidade de reaprender como interagir com ele; 4) Baixa taxa de erros: nos sistemas que possuem este atributo os usuários cometem poucos erros ao usá-lo e, quando cometem, sua correção é simples; 5) Satisfação: o sistema deve ser agradável de usar, de forma que os usuários sintam-se subjetivamente satisfeitos ao utilizá-lo.

\subsection{Arquitetura da Informação}

A Arquitetura da Informação teria o papel de solucionar o que era complexo e de arrumar a imensa quantidade de dados do mundo civilizado (BUSTAMANTE, 2002).

(ROSENFELD E MORVILLE, 2006) apresentam quatro definições para arquitetura da informação: 1) "é o projeto estrutural de ambientes de informação compartilhados"; 2) "é a combinação de sistemas de organização, rotulagem, busca e navegação dentro de websites e intranets"; 3) é "a arte e a ciência que dá forma a produtos de informação e experiências para apoio a usabilidade e facilidade de busca (findability), também conhecido como "encontrabilidade"; 4) e por último, é "uma disciplina emergente e comunidade de prática focada em trazer princípios de design e arquitetura para o panorama digital".

Destaca-se que a Arquitetura da Informação está preocupada com a forma como as pessoas processam cognitivamente a informação. Ela é percebida em qualquer sistema que exija que os usuários entendam as informações apresentadas (GARRETT, 2010). 


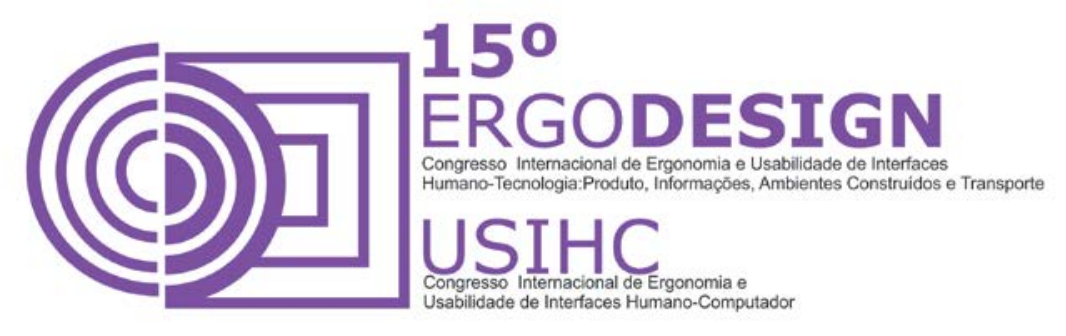

Arquitetura da Informação refere-se "a tarefa de criar, mapear e construir, tornando as informações claramente identificáveis e sua distribuição bem definida" (RODRIGUES, 2001).

O problema principal dos websites é que as pessoas não encontram o que precisam, e acabam desistindo da tarefa. "Há muitos casos em que isso acontece não apenas por problemas de usabilidade, mas por falhas em sua arquitetura da informação". O papel do arquiteto da informação, é portanto o de "construir estradas da informação, atalhos, pontes e conexões com o intuito de permitir o acesso mais rápido e intuitivo à informação" (SANTA ROSA E MORAES, 2012a).

De acordo com (ROSENFELD E MORVILLE, 2006) a Arquitetura da Informação de um website está dividida em quatro grandes sistemas, cada um composto por regras e aplicações. Juntos eles reúnem todos os elementos de interação do usuário com a informação apresentada pelo website. São eles: 1) Sistema de Organização (Organization System): Define a classificação de todo o conteúdo; 2) Sistema de Rotulação (Labeling System): Estabelece as formas de representação, de apresentação, da informação definindo rótulos para cada elemento informativo; 3) Sistema de Navegação (Navegation System): Especifica as maneiras de navegar, de se mover pelo espaço informacional e hipertextual; e 4) Sistema de Busca (Search System): Determina as perguntas que o usuário pode fazer e o conjunto de respostas que irá obter.

\section{MÉTODOS E TÉCNICAS}

\subsection{Cardsorting}

Cardsorting é um método de prototipagem rápido, barato e seguro utilizado pela Arquitetura da Informação, que consiste em entregar ao usuário alguns cartões, cada um deles representando um conteúdo do sistema, e solicitar que ele (usuário) os organize em rótulos, formando categorias.

Cardsorting é uma técnica de arranjo de cartões na qual cinco participantes, em média, realizam a atividade de ordenação, grupamento, hierarquização, nomeação e classificação de dados abstratos, arbitrários ou não, correspondentes aos itens de conteúdo de um sistema (SANTA ROSA E MORAES, 2012b).

O objetivo é gerar uma estrutura global da informação ou avaliar uma estrutura existente, aumentando a probabilidade do usuário encontrar informações enquanto estiver navegando. $\mathrm{O}$ Cardsorting permite entender como os usuários reais categorizam as informações disponíveis, identificando qual terminologia é a mais usual, qual pode gerar confusões e que termos são mais difíceis de categorizar. Ao contar com a participação direta dos usuários finais, 0 Cardsorting pode ajudar a cortar caminhos tediosos ou debates infrutíferos (MAURER E WARFEL, 2004).

O Cardsorting possui duas variantes básicas: as formas aberta e fechada. Na forma aberta os participantes recebem cartões em branco e é mais usada para levantamento/descoberta de termos. Na forma fechada os cartões já estão rotulados sendo a forma mais utilizada para 


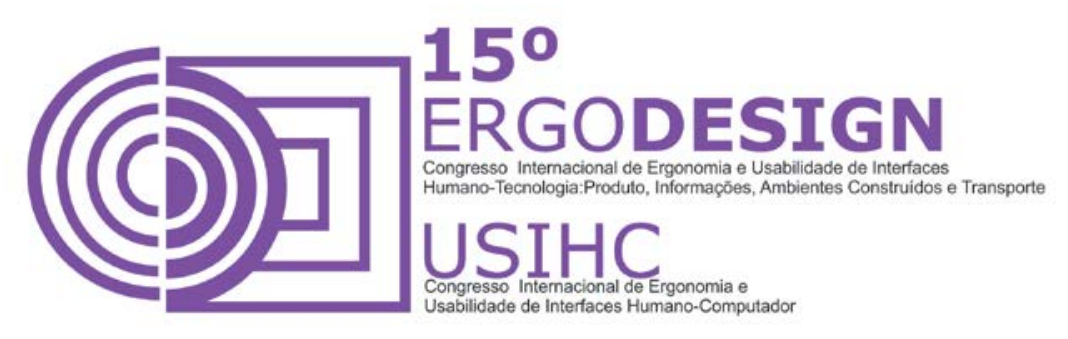

validação de estruturas e de uso mais comum. Entre essas duas opções pode-se utilizar formas híbridas, mais fechadas ou mais abertas, balanceadas de acordo com as necessidades de cada projeto (ROSENFELD E MORVILLE, 2006).

A aplicação do método Cardsorting foi realizada com seis participantes que foram previamente convidados e se disponibilizaram a colaborar com a aplicação do método, sendo estes bibliotecária, professor, administradora, secretária, jornalista e aluno da Pós-Graduação.

A atividade teve como objetivo a reformulação do menu atual do site do Instituto do Cérebro e teve uma duração de aproximadamente cinquenta minutos.

A aplicação da técnica Cardsorting se deu de maneira aberto-fechada. Aberta, para que novos menus ou conteúdos dos menus pudessem ser incluídos. E fechada, pois todos os menus e os conteúdos de cada menu foram descritos em cartões tipo post it e espalhados em cima de uma mesa (Figura 1) para que os participantes pudessem discutir e relacionar esses cartões.

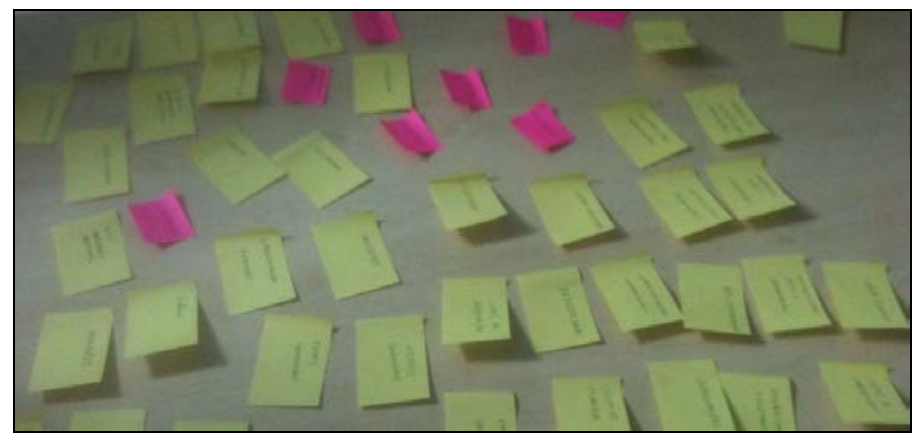

Figura 1. Aplicação da Técnica Cardosrting (Fonte: Elaborada pelo autor).

\section{RESULTADOS}

\subsection{Resultados da aplicação da Técnica Cardsorting}

A aplicação da técnica Cardsorting contou com a colaboração de um grupo de seis participantes (bibliotecária, professor, administradora, secretária, jornalista e aluno) e teve como objetivo a reformulação do menu atual do site do Instituto do Cérebro. 

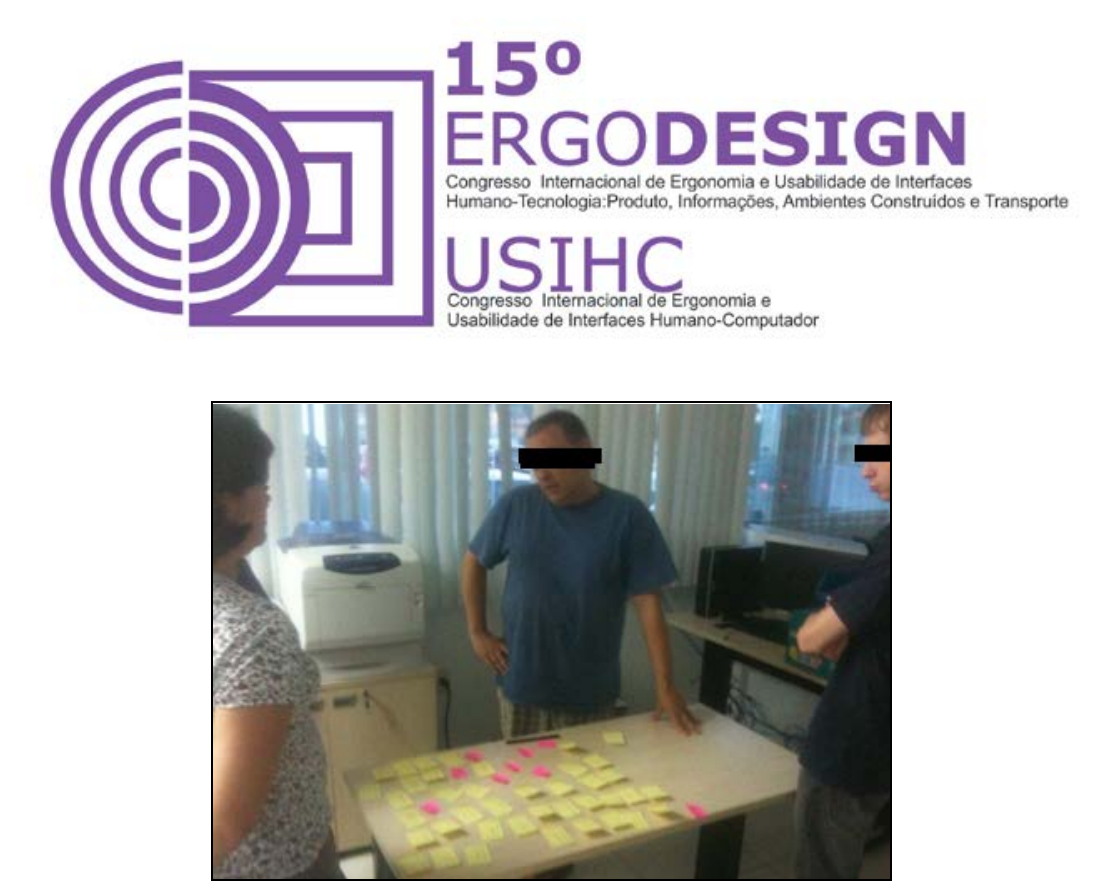

Figura 2. Aplicação da Técnica Cardsorting (Fonte: Elaborada pelo autor).

O Cardsorting foi aplicado de maneira aberto-fechada e teve uma duração de aproximadamente cinquenta minutos. A forma aberta permitiu que novos cartões tipo post it com sugestões de novos menus ou conteúdos dos menus fossem sendo incluídos ao decorrer das discussões do grupo. E fechada, pois todos os menus e os conteúdos de cada menu já existentes, foram descritos em cartões tipo post it e espalhados em cima de uma mesa (Figura 2) para que os participantes pudessem discutir e relacionar esses cartões.

Inicialmente foi explicado o objetivo da aplicação da técnica Cardsorting e como funcionava. Os participantes foram informados que todo o processo seria gravado em áudio, fotografado e que também seriam feitas algumas anotações.

O mediador espalhou os cartões tipo post it em cima de uma mesa e um dos participantes falou que na sua opinião o menu do site deveria ser enxugado, reduzindo os menus desnecessários e integrando os itens que seriam mantidos em outros menus.

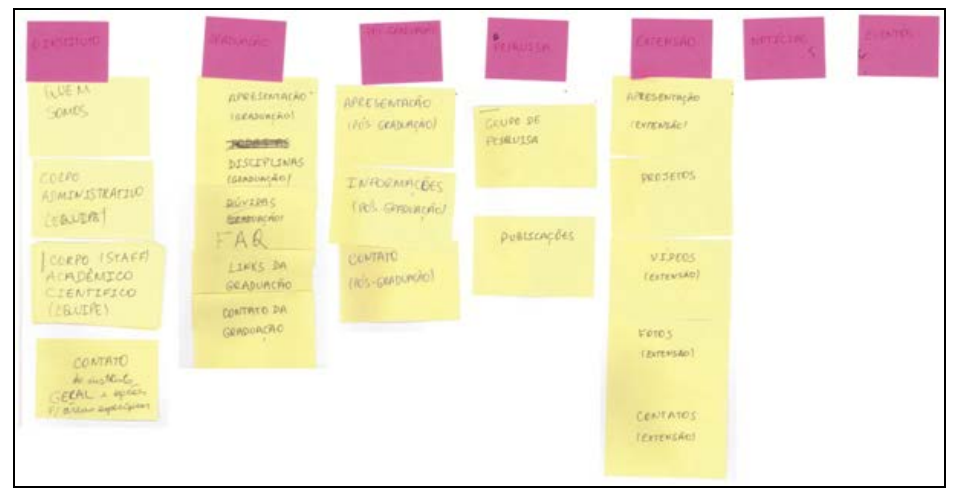

Figura 3. Aplicação da técnica Cardsorting (Fonte: Elaborada pelo autor).

Para diferenciar o menu principal dos submenus e itens dos menus foram utilizados cartões (post it) nas corres rosa e amarelo. Após isso, os participantes começaram a pegar os cartões na mesa e agrupá-los em categorias (Figura 3) que foram sendo modificadas durante 0 


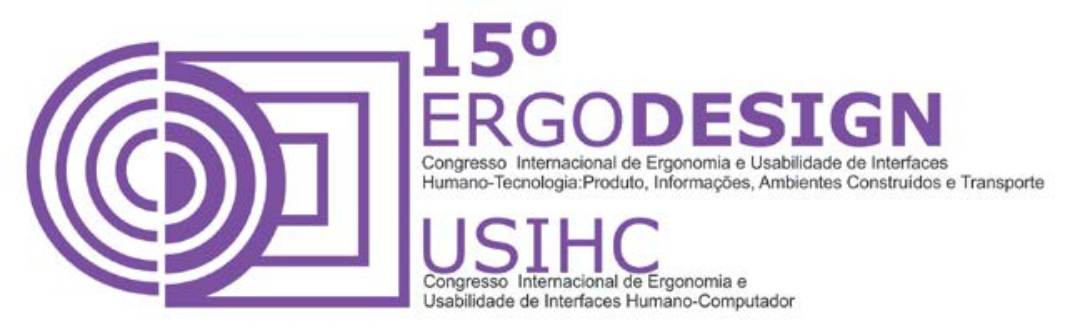

processo. Algumas vezes ficava decidido pelos participantes da técnica Cardsorting, que um determinado item do menu seria excluído ou teria a nomenclatura modificada, e em seguida, esse item de menu ou nomenclatura, já discutido anteriormente, voltava a discussão e era realocado para outra categoria.

\begin{tabular}{|lllllll|}
\hline OINSTITUTD PESCUISA & ENSINO & EXTENSAO & NOTICIAS & EVENTOS & OPORTUNIDADES & FERRAMENTAS JVRES \\
\hline
\end{tabular}

Figura 4. Menu principal do site do Instituto do Cérebro (Fonte: Elaborada pelo autor).

A figura 4 ilustra o menu principal do site atual do Instituto do Cérebro atualmente. Durante a aplicação da técnica Cardsorting os participantes sugeriram algumas modificações.

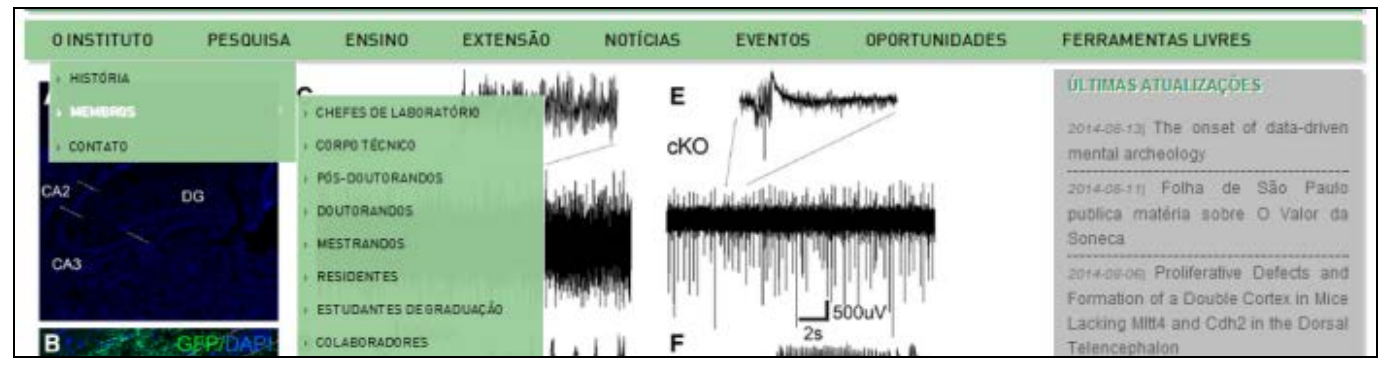

Figura 5. Menu ‘O Instituto’ (Fonte: Elaborada pelo autor).

Todos os participantes concordaram que o menu 'O Instituto' (Figura 5) é relevante e que deve permanecer, inclusive com a mesma nomenclatura. Mas, foram sugeridas algumas modificações nos itens do menu 'O Instituto'.

Na visão do grupo a nomenclatura do item 'História' deve ser alterada para 'Quem somos', que parece ser um termo mais adequado para atrair o usuário para conhecer quem é o Instituto do Cérebro.

O submenu 'Membros' foi bastante discutido, se chegando a um consenso que deve ser excluído, e criadas duas novas categorias que são 'Equipe Administrativa' e 'Equipe Acadêmico-Científica'.

Farão parte da categoria 'Equipe Administrativa', os funcionários do Instituto que desempenham atividades administrativas (secretárias, administradora, etc.). Para o professor Martin, 'Corpo Técnico' são as pessoas técnico-científicas. Então, o mesmo sugeriu excluir o item 'Corpo Técnico' e criar as novas categorias. Sugestão esta que foi bastante discutida e bem aceita por todos os participantes.

O submenu 'Equipe Acadêmico-Científica', englobará as categorias 'Professores', 'Pósdoutorandos', 'Doutorandos', 'Mestrandos', 'Técnicos', 'Iniciação Científica', 'Colaboradores' e 'Visitantes' (categoria que anteriormente estava agrupada no menu 'Pesquisa' e se denominava 'Pesquisador Visitante'). 


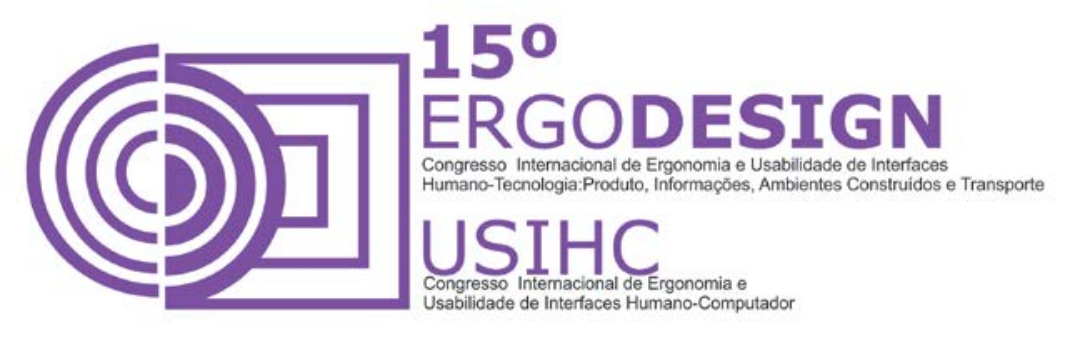

Na categoria 'Técnicos', serão incluídos os funcionários que desempenham atividades técnicas (veterinária, técnicos de laboratórios, etc.), sejam eles efetivos da UFRN ou terceirizados. Atualmente, os funcionários efetivos da UFRN, sejam os administrativos ou técnicos estão incluídos na categoria 'Corpo Técnico' e os funcionários terceirizados não são mostrados no site.

A categoria 'Residentes' deve ser excluída, pois o Instituto do Cérebro não possui um programa de residência.

Foi sugerido, ainda, que no formulário da página de Contato, que pode ser acessada através do menu 'O instituto', tenha uma caixa de seleção com todos os contatos dos setores do Instituto do Cérebro, para que o usuário possa escolher o setor que deseja enviar sua mensagem.

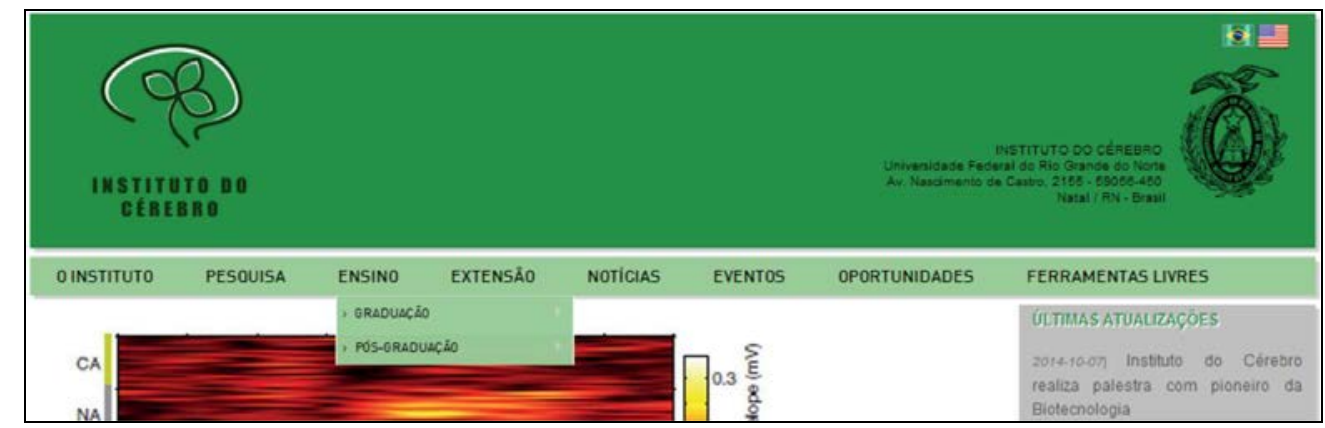

Figura 6. Menu 'Ensino' (Fonte: Elaborada pelo autor)

A figura 6 ilustra o menu 'Ensino', que atualmente engloba os submenus 'Graduação' e 'PósGraduação'. Os participantes sugeriram remover o menu 'Ensino' e incluir as opções 'Graduação' e 'Pós-Graduação' diretamente no menu principal, diminuindo um clique para acessar essas opções.

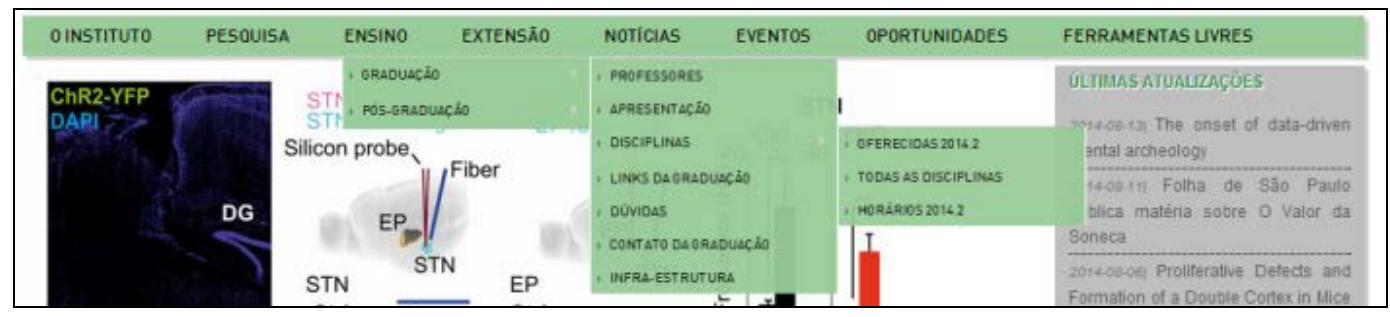

Figura 7. Opção ‘Graduação’ no menu ‘Ensino’ (Fonte: Elaborada pelo autor).

Um dos participantes sugeriu remover do site o menu 'Graduação' (Figura 7), mas outros participantes explicaram que existe o eixo temático da Graduação em Neurociências, por isso, a importância de manter informações sobre a Graduação no site. Então, o grupo de participantes optou por mover opção 'Graduação', que antes era um submenu da opção 'Ensino', para o menu principal do site do Instituto do Cérebro. 


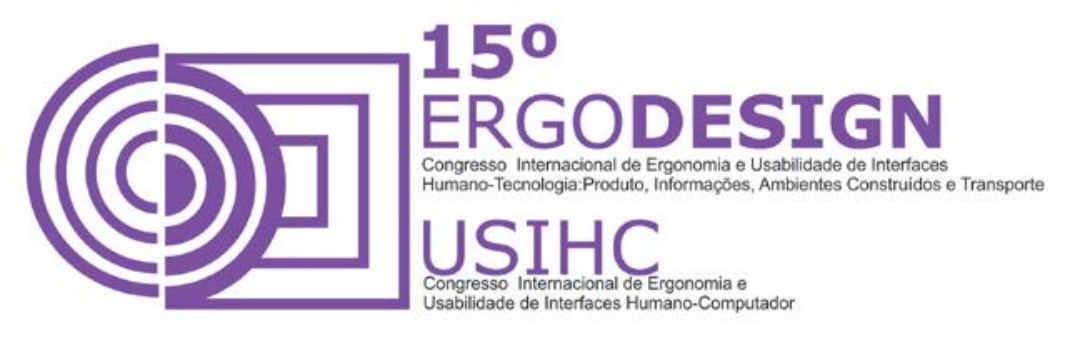

Sugeriram também, remover 'Professores', visto que os professores já são exibidos no menu 'O Instituto'.

Para o item 'Apresentação', a sugestão foi manter esta opção no menu 'Graduação', e ainda, incluir na página de 'Apresentação' os contatos da graduação e infraestrutura, eliminando assim, as opções 'Contato da Graduação' e 'Infraestrutura'. Foi sugerido, também, incluir os contatos da graduação na página de contato geral no menu 'O Instituto'.

Quanto aos itens do submenu 'Disciplinas', as opções 'Oferecidas', 'Todas as disciplinas' e 'Horários', foi sugerido concentrar todas as informações em 'Disciplinas', criando uma única página, com uma estrutura que permita o usuário visualizar as disciplinas oferecidas no semestre com seus horários, e ainda, todas as disciplinas da grade curricular com as informações dos períodos em que a disciplina foi oferecida. Com isso, seriam eliminados alguns cliques e tornaria o menu 'Graduação' mais objetivo.

A opção 'Links da graduação' deve permanecer dentro do menu "Graduação", mas a nomenclatura deve ser alterada para 'Links Úteis', e que o layout da página seja melhorado, para que fique mais atrativo.

E por fim, foi sugerido alterar a nomenclatura da opção 'Dúvidas da graduação' para 'Perguntas frequentes'.

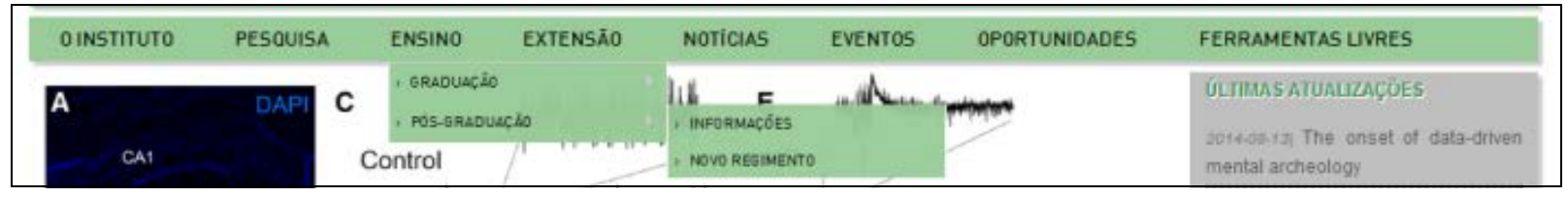

Figura 8. Opção 'Pós-Graduação' do menu ‘Ensino' (Fonte: Elaborada pelo autor).

O submenu 'Pós-Graduação' (Figura 8), deve ser movido para o menu principal. Foi sugerido criar uma página 'Apresentação' para a Pós-Graduação.

\begin{tabular}{|c|c|c|c|c|c|c|c|}
\hline OINSTITUTO & PESQUISA & ENSINO & EXTENSÃOO & NOTICIAS & EVENTOS & OPORTUNIDADES & FERRAMENTAS LUVES \\
\hline $\begin{array}{l}\text { A } \\
\quad 30 \mid\end{array}$ & 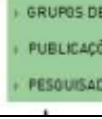 & UTSA & & B & oh 1 (Rat & PRE WKI & 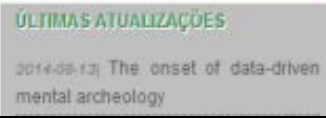 \\
\hline
\end{tabular}

Figura 9. Menu 'Pesquisa' (Fonte: Elaborada pelo autor).

Os menus 'Pesquisa' (Figura 9) e 'Extensão' devem permanecer no menu principal por fazerem parte do tripé Ensino, Pesquisa e Extensão. Mas, o menu 'Pesquisa' que antes era a segunda opção do menu, foi movido para depois de Pós-Graduação (quarta opção do menu).

Não foi feita nenhuma alteração nos itens 'Grupos de Pesquisa' e 'Publicações', que permaneceram dentro do menu 'Pesquisa'.

A categoria 'Pesquisador Visitante', deve ser removida do menu 'Pesquisa' e incluída no submenu 'Equipe Acadêmico-Científica' do menu 'O Instituto', e ainda, alterada sua nomenclatura para 'Visitante'. 

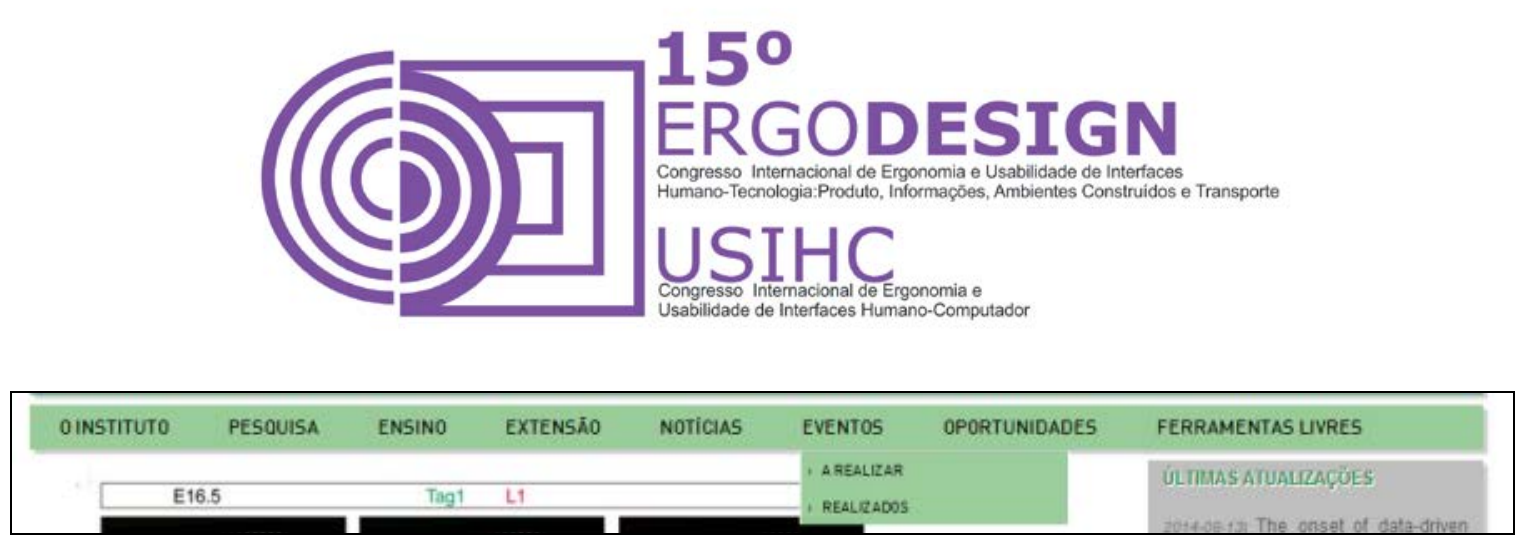

Figura 10. Menu 'Eventos' (Fonte: Elaborada pelo autor).

No menu 'Eventos' (Figura 10) foi sugerido remover as categorias 'A realizar' e 'Realizados', e que seja criada uma na nova página de eventos com um calendário, no qual o usuário possa visualizar um evento clicando no mês desejado.

\begin{tabular}{|c|c|c|c|c|c|c|c|}
\hline OINSTITUTO & PESOUISA & ENSINO & EXTENSĀO & NOTICIAS & EVENTOS & OPORTUNIDADES & FERRAMENTAS UVRES \\
\hline & DAPI & & whending & $\mathbf{E}$ & & 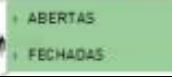 & IAS ATUALIZACOEES \\
\hline
\end{tabular}

Figura 11. Menu 'Oportunidades' (Fonte: Elaborada pelo autor).

Atualmente, o menu 'Oportunidades' (Figura 11), permite o usuário acessar uma página de divulgação de vagas de estágio, vagas para bolsistas, concursos, entre outras oportunidades.

A maioria dos participantes entraram em um consenso que o menu 'Oportunidades' deve ser removido do site. Na visão do grupo, não é necessário as oportunidades estarem fixas no menu. Quando existir uma oportunidade, essa deve ser divulgada por meio de notícias.

Outra opção sugerida por um dos participantes, que inicialmente não concordou com o pensamento de excluir a página de oportunidades, foi que 'Oportunidades' fossem realocadas para o menu 'O Instituto'.

\begin{tabular}{|c|c|c|c|c|c|c|c|c|}
\hline OINSTITUTO & PESQUISA & ENSINO & EXTENSǞO & NOTICIAS & EVENTOS & OPORTUNIDADES & FERRAMENTAS LIVRES & \\
\hline CA1 & DAPI & Ontrol & Whit & $\begin{array}{l}\mathbf{E} \\
\text { cKO }\end{array}$ & & & 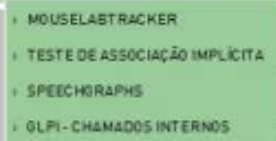 & Iriven \\
\hline
\end{tabular}

Figura 12. Menu 'Ferramentas Livres' (Fonte: Elaborada pelo autor).

O grupo sugeriu remover o menu 'Ferramentas Livres' (Figura 12) e realocar os softwares desenvolvidos pelo Instituto do Cérebro na página do grupo de pesquisa que os desenvolveu. As demais opções foram consideradas desnecessárias por parte do grupo que participou da técnica Cardsorting para definição do novo menu.

Na opinião do grupo os itens 'Teste de Associação Implícita' e 'GLPI - Chamados Internos', devem ser removidos.

Quanto aos softwares 'SpeechGraphs' e 'MouseLabTracker', a sugestão do grupo foi manter essas duas páginas nos respectivos grupos de pesquisa do responsável pela ferramenta.

A Figura 13 ilustra através de um mapa, como é a estrutura atual do menu do site do Instituto do Cérebro após a aplicação da técnica Cardsorting. 

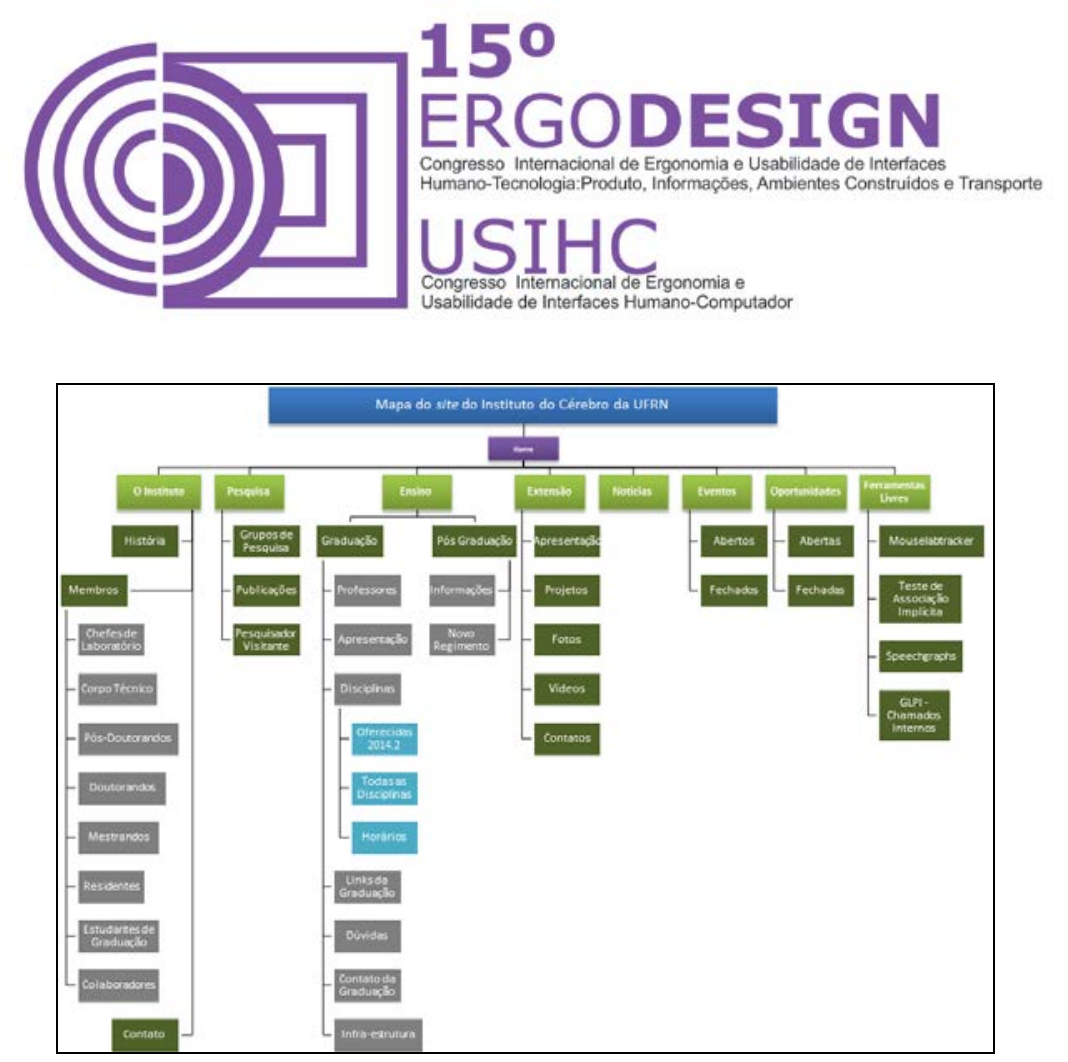

Figura 13. Organização atual do menu do site do Instituto do Cérebro (Fonte: Elaborado pelo autor). A figura 14 ilustra através de um mapa, o resultado da organização do conteúdo do menu do site do Instituto do Cérebro após a aplicação da técnica Cardsorting.

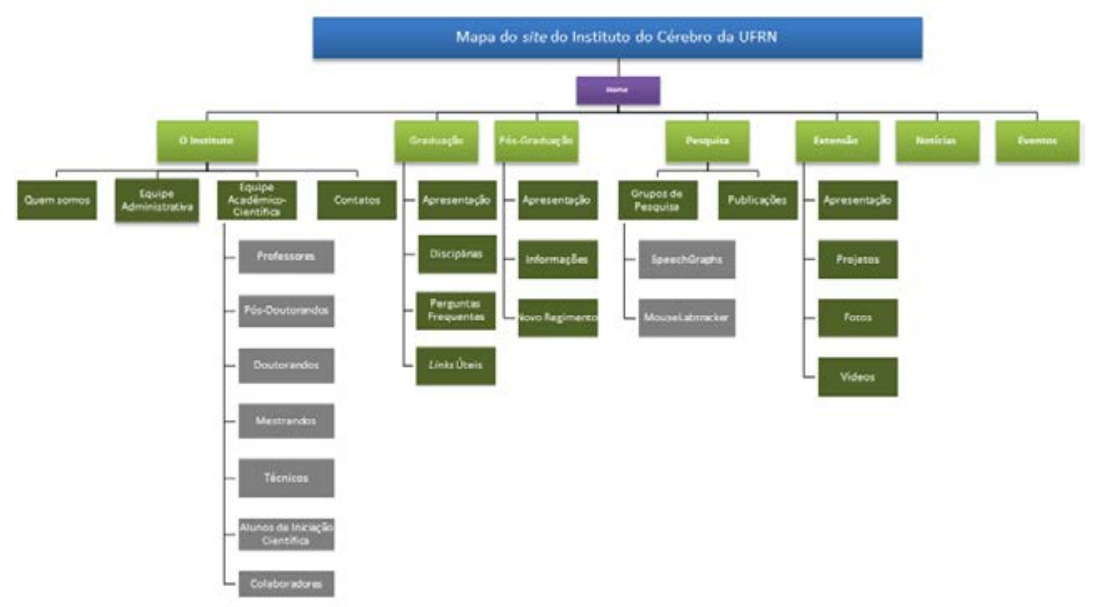

Figura 14. Organização do menu do site do I Ce após a aplicação da técnica Cardsorting (Fonte: Elaborado pelo autor).

\section{CONCLUSÃO}

A aplicação do método Cardsorting é uma alternativa fácil e de baixo custo que permitiu verificar padrões de comportamento no processo classificatório dos usuários, e ainda, identificar quais 


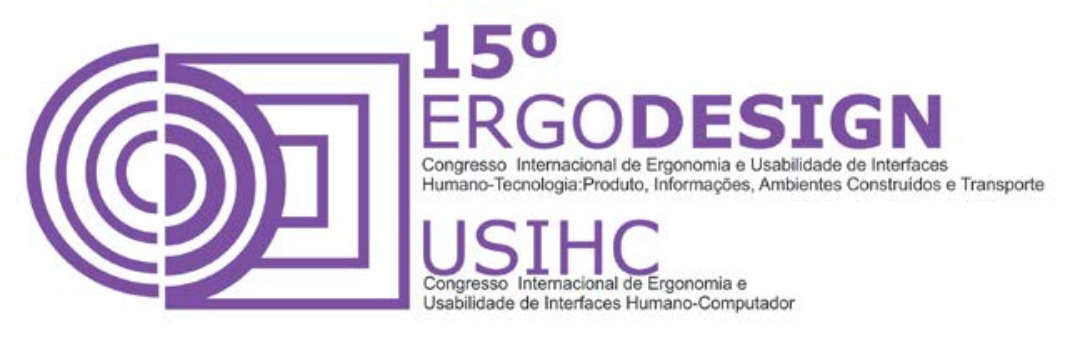

grupamentos e terminologias são mais usuais, quais podem gerar confusões e que termos são mais difíceis de categorizar.

De acordo com a visão do grupo de colaboradores, e por meio da aplicação do método Cardsorting, será necessário realizar algumas alterações no menu do site do Instituto do Cérebro da UFRN. Recomenda-se excluir alguns dos conteúdos existentes e alterar a nomenclatura de outros, bem como, incluir novos itens e menus.

Conclui-se, portanto, por meio da análise dos dados obtidos a partir da aplicação do método Cardsorting, foram verificados problemas relacionados a rotulagem de alguns termos do menu, e ainda, ausência e posicionamento inadequado de informações que seriam de alta relevância para os usuários. O menu do site do Instituto do Cérebro da UFRN necessita ser reformulado com base nos problemas relatados pelos usuários.

\section{REFERÊNCIAS BIBLIOGRÁFICAS}

ASSOCIAÇÃO BRASILEIRA DE NORMAS TÉCNICAS. NBR 9241-11: Requisitos Ergonômicos para Trabalho de Escritórios com Computadores Parte 11 - Orientações sobre Usabilidade, 2002. Disponível em: <http://www.inf.ufsc.br/ cybis/pg2003/iso9241-11F2.pdf>. Acesso em: 12 out. 2013.

BUSTAMANTE, J. La arquitectura de la información del siglo XX al XXI. The Information Architecture Institute, Diciembre 18, 2002. Disponível em: <http://iainstitute.org/pt/translations/000330.html>. Acesso em: 20 jan. 2014.

CYBIS, W.; BETIOL, A. H.; FAUST, R. Ergonomia e usabilidade: conhecimentos, métodos e aplicações. 2. ed. São Paulo: Novatec Editora, 2010.

GARRETT, J. J. The elements of user experience. 2. ed. New York: New Rides, 2010.

ICE. História. 2011. Disponível em: <http://neuro.ufrn.br/instituto/historia>. Acesso em: 05 jun. 2014.

Site. Disponível em: <http://neuro.ufrn.br>. Acesso em: 05 mar. 2014.

MAURER, D.; WARFEL, T. Card sorting: a definitive guide. 2004. Disponível em:

<http://boxesandarrows.com/card-sorting-a-definitive-guide/>. Acesso em: 12 set. 2012.

NIELSEN, J. Usability Engineering. Montview: Sunsoft, 1993.

RODRIGUES, B. Não à arquitetura da embromação. Webinsider, fev. 2001. Disponível em:

<http://webinsider.com.br/2001/02/22/nao-a-arquitetura-da-embromacao/>. Acesso em: 12 fev. 2014.

ROSENFELD, L.; MORVILLE, P. Information Architecture for the Word Wide Web. 3ed. Sebastopol: O'Reilly, 2006.

SANTA ROSA, J. G.; MORAES, A. Avaliação e projeto no design de interfaces. 2.ed. Teresópolis, RJ: 2AB, 2012. 


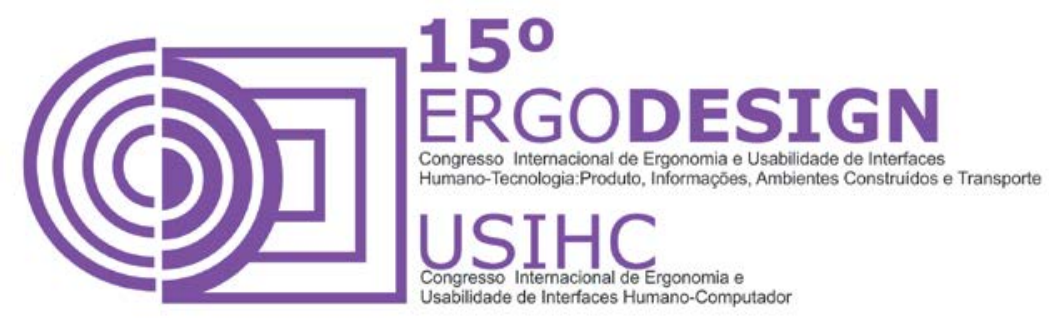

Design Participativo. Rio de Janeiro, RJ: Rio Book's, 2012b. 